\title{
Complete Convergence for Arrays of Rowwise Asymptotically Almost Negatively Associated Random Variables
}

\author{
Xuejun Wang, Shuhe Hu, and Wenzhi Yang \\ School of Mathematical Science, Anhui University, Hefei 230039, China \\ Correspondence should be addressed to Xuejun Wang, 07019@ahu.edu.cn
}

Received 24 April 2011; Revised 3 September 2011; Accepted 18 September 2011

Academic Editor: Wei-Der Chang

Copyright (C) 2011 Xuejun Wang et al. This is an open access article distributed under the Creative Commons Attribution License, which permits unrestricted use, distribution, and reproduction in any medium, provided the original work is properly cited.

Let $\left\{X_{n i}, i \geq 1, n \geq 1\right\}$ be an array of rowwise asymptotically almost negatively associated random variables. Some sufficient conditions for complete convergence for arrays of rowwise asymptotically almost negatively associated random variables are presented without assumptions of identical distribution. As an application, the Marcinkiewicz-Zygmund type strong law of large numbers for weighted sums of asymptotically almost negatively associated random variables is obtained.

\section{Introduction}

The concept of complete convergence was introduced by Hsu and Robbins [1] as follows. A sequence of random variables $\left\{U_{n}, n \geq 1\right\}$ is said to converge completely to a constant $C$ if $\sum_{n=1}^{\infty} P\left(\left|U_{n}-C\right|>\varepsilon\right)<\infty$ for all $\varepsilon>0$. In view of the Borel-Cantelli lemma, this implies that $U_{n} \rightarrow C$ almost surely (a.s.). The converse is true if the $\left\{U_{n}, n \geq 1\right\}$ are independent. Hsu and Robbins [1] proved that the sequence of arithmetic means of independent and identically distributed (i.i.d.) random variables converges completely to the expected value if the variance of the summands is finite. Since then many authors studied the complete convergence for partial sums and weighted sums of random variables. The main purpose of the present investigation is to provide the complete convergence results for weighted sums of asymptotically almost negatively associated random variables and arrays of rowwise asymptotically almost negatively associated random variables.

Firstly, let us recall the definitions of negatively associated and asymptotically almost negatively associated random variables. 
Definition 1.1. A finite collection of random variables $X_{1}, X_{2}, \ldots, X_{n}$ is said to be negatively associated (NA, in short) if for every pair of disjoint subsets $A_{1}, A_{2}$ of $\{1,2, \ldots, n\}$,

$$
\operatorname{Cov}\left\{f\left(X_{i}: i \in A_{1}\right), g\left(X_{j}: j \in A_{2}\right)\right\} \leq 0,
$$

whenever $f$ and $g$ are coordinatewise nondecreasing such that this covariance exists. An infinite sequence $\left\{X_{n}, n \geq 1\right\}$ is NA if every finite subcollection is negatively associated.

An array of random variables $\left\{X_{n i}, i \geq 1, n \geq 1\right\}$ is called rowwise NA random variables if, for every $n \geq 1,\left\{X_{n i}, i \geq 1\right\}$ is a sequence of NA random variables.

The concept of negative association was introduced by Joag-Dev and Proschan [2]. By inspecting the proof of maximal inequality for the NA random variables in Matula [3], one also can allow negative correlations provided they are small. Primarily motivated by this, Chandra and Ghosal $[4,5]$ introduced the following dependence.

Definition 1.2. A sequence $\left\{X_{n}, n \geq 1\right\}$ of random variables is called asymptotically almost negatively associated (AANA, in short) if there exists a nonnegative sequence $q(n) \rightarrow 0$ as $n \rightarrow \infty$ such that

$$
\operatorname{Cov}\left(f\left(X_{n}\right), g\left(X_{n+1}, X_{n+2}, \ldots, X_{n+k}\right)\right) \leq q(n)\left[\operatorname{Var}\left(f\left(X_{n}\right)\right) \operatorname{Var}\left(g\left(X_{n+1}, X_{n+2}, \ldots, X_{n+k}\right)\right)\right]^{1 / 2},
$$

for all $n, k \geq 1$ and for all coordinatewise nondecreasing continuous functions $f$ and $g$ whenever the variances exist.

An array of random variables $\left\{X_{n i}, i \geq 1, n \geq 1\right\}$ is called rowwise AANA random variables if, for every $n \geq 1,\left\{X_{n i}, i \geq 1\right\}$ is a sequence of AANA random variables.

The family of AANA sequence contains NA (in particular, independent) sequences (with $q(n)=0, n \geq 1$ ) and some more sequences of random variables which are not much deviated from being negatively associated. An example of an AANA sequence which is not NA was constructed by Chandra and Ghosal [4].

Since the concept of AANA sequence was introduced by Chandra and Ghosal [4], many applications have been found. See, for example, Chandra and Ghosal [4] derived the Kolmogorov type inequality and the strong law of large numbers of MarcinkiewiczZygmund, Chandra and Ghosal [5] obtained the almost sure convergence of weighted averages, Ko et al. [6] studied the Hájek-Rényi type inequality, Wang et al. [7] established the law of the iterated logarithm for product sums, Yuan and An [8] established some Rosenthal type inequalities for maximum partial sums of AANA sequence, and Wang et al. [9] obtained some strong growth rate and the integrability of supremum for the partial sums of AANA random variables, and so forth.

Our goal in this paper is to study the complete convergence for arrays of rowwise AANA random variables under some moment conditions. As an application, the Marcinkiewicz-Zygmund type strong law of large numbers for weighted sums of AANA random variables is obtained. We will give some sufficient conditions for complete convergence for an array of rowwise AANA random variables without assumption of identical distribution. The results presented in this paper are obtained by using the truncated method and the classical maximal type inequality of AANA random variables (Lemma 1.5 below). 
Throughout the paper, let $\left\{X_{n i}: i \geq 1, n \geq 1\right\}$ be an array of rowwise AANA random variables with the mixing coefficients $\{q(i), i \geq 1\}$ in each row. For $p>1$, let $q \doteq p /(p-1)$ be the dual number of $p$. Let $I(A)$ be the indicator function of the set $A$. $C$ denotes a positive constant which may be different in various places and $a_{n}=O\left(b_{n}\right)$ stands for $a_{n} \leq C b_{n}$.

Definition 1.3. An array of random variables $\left\{X_{n i}, i \geq 1, n \geq 1\right\}$ is said to be stochastically dominated by a random variable $X$ if there exists a positive constant $C$ such that

$$
P\left(\left|X_{n i}\right|>x\right) \leq C P(|X|>x)
$$

for all $x \geq 0, i \geq 1$ and $n \geq 1$.

The following lemmas are useful for the proofs of the main results.

Lemma 1.4 (cf. Yuan and An [8, Lemma 2.1]). Let $\left\{X_{n}, n \geq 1\right\}$ be a sequence of AANA random variables with mixing coefficients $\{q(n), n \geq 1\}$, let $f_{1}, f_{2}, \ldots$ be all nondecreasing (or all nonincreasing) functions, then $\left\{f_{n}\left(X_{n}\right), n \geq 1\right\}$ is still a sequence of AANA random variables with mixing coefficients $\{q(n), n \geq 1\}$.

Lemma 1.5 (cf. Yuan and An [8, Theorem 2.1]). Let $\left\{X_{n}, n \geq 1\right\}$ be a sequence of AANA random variables with $E X_{i}=0$ for all $i \geq 1$ and $p \in\left(3 \cdot 2^{k-1}, 4 \cdot 2^{k-1}\right]$, where integer number $k \geq 1$. If $\sum_{n=1}^{\infty} q^{q / p}(n)<\infty$, then there exists a positive constant $D_{p}$ depending only on $p$ such that for all $n \geq 1$,

$$
E\left(\max _{1 \leq j \leq n}\left|\sum_{i=1}^{j} X_{i}\right|^{p}\right) \leq D_{p}\left\{\sum_{i=1}^{n} E\left|X_{i}\right|^{p}+\left(\sum_{i=1}^{n} E X_{i}^{2}\right)^{p / 2}\right\}
$$

Lemma 1.6. Let $\left\{X_{n}, n \geq 1\right\}$ be a sequence of random variables which is stochastically dominated by a random variable $X$. For any $\alpha>0$ and $b>0$, the following two statements hold:

$$
\begin{gathered}
E\left|X_{n}\right|^{\alpha} I\left(\left|X_{n}\right| \leq b\right) \leq C_{1}\left[E|X|^{\alpha} I(|X| \leq b)+b^{\alpha} P(|X|>b)\right], \\
E\left|X_{n}\right|^{\alpha} I\left(\left|X_{n}\right|>b\right) \leq C_{2} E|X|^{\alpha} I(|X|>b),
\end{gathered}
$$

where $C_{1}$ and $C_{2}$ are positive constants.

\section{Main Results}

Let $\left\{X_{n i}: i \geq 1, n \geq 1\right\}$ be an array of rowwise AANA random variables with the mixing coefficients $\{q(i), i \geq 1\}$ in each row, and let $\left\{a_{n i}: i \geq 1, n \geq 1\right\}$ be an array of real numbers. Let $\left\{X_{i}, i \geq 1\right\}$ be a sequence of AANA random variables with the mixing coefficients $\{q(i), i \geq 1\}$ and let $\left\{a_{i}, i \geq 1\right\}$ be a sequence of real numbers. We consider the following conditions.

$\left(\mathrm{H}_{1}\right)$ There exist some $\delta$ with $0<\delta<1$ and some $\alpha$ with $0<\alpha<2$ such that $\sum_{i=1}^{n}\left|a_{n i}\right|^{\alpha}=$ $O\left(n^{\delta}\right)$, and assume further that $E X_{n i}=0$ if $1<\alpha<2$.

$\left(\mathrm{H}_{2}\right)$ There exists some $p \in\left(3 \cdot 2^{k-1}, 4 \cdot 2^{k-1}\right]$ such that $\sum_{i=1}^{\infty} q^{q / p}(i)<\infty$, where integer number $k \geq 1$. 
$\left(\mathrm{H}_{3}\right)$ For some $h>0$ and $\gamma>0$,

$$
E \exp \left(h|X|^{\gamma}\right)<\infty
$$

$\left(\mathrm{H}_{4}\right)$ There exist some $\delta$ with $0<\delta<1$ and some $\alpha$ with $0<\alpha<2$ such that $\sum_{i=1}^{n}\left|a_{i}\right|^{\alpha}=$ $O\left(n^{\delta}\right)$, and assume further that $E X_{n}=0$ if $1<\alpha<2$.

$\left(\mathrm{H}_{5}\right)$ There exists some $\alpha$ with $0<\alpha<2$ such that $\sum_{i=1}^{n}\left|a_{n i}\right|^{\alpha}=O(n)$ and assume further that $E X_{n i}=0$ if $1<\alpha<2$.

$\left(\mathrm{H}_{6}\right)$ There exists some $\alpha$ with $0<\alpha<2$ such that $\sum_{i=1}^{n}\left|a_{i}\right|^{\alpha}=O(n)$ and assume further that $E X_{n}=0$ if $1<\alpha<2$.

Our main results are as follows.

Theorem 2.1. Let $\left\{X_{n i}: i \geq 1, n \geq 1\right\}$ be an array of rowwise AANA random variables which is stochastically dominated by a random variable $X$, and let $\left\{a_{n i}: i \geq 1, n \geq 1\right\}$ be an array of real numbers. Suppose that the conditions $\left(H_{1}\right)-\left(H_{3}\right)$ are satisfied. Then, for any $\varepsilon>0$,

$$
\sum_{n=1}^{\infty} n^{s \alpha-2} P\left(\max _{1 \leq j \leq n}\left|\sum_{i=1}^{j} a_{n i} X_{n i}\right|>\varepsilon b_{n}\right)<\infty,
$$

where $s \geq 1 / \alpha$ and $b_{n} \doteq n^{1 / \alpha} \log ^{1 / \gamma} n$.

Proof. For fixed $n \geq 1$, define

$$
\begin{gathered}
X_{i}^{(n)}=-b_{n} I\left(X_{n i}<-b_{n}\right)+X_{n i} I\left(\left|X_{n i}\right| \leq b_{n}\right)+b_{n} I\left(X_{n i}>b_{n}\right), \quad i \geq 1 \\
T_{j}^{(n)}=\sum_{i=1}^{j} a_{n i}\left(X_{i}^{(n)}-E X_{i}^{(n)}\right), \quad j=1,2, \ldots, n .
\end{gathered}
$$

It is easy to check that for any $\varepsilon>0$,

$$
\left(\max _{1 \leq j \leq n}\left|\sum_{i=1}^{j} a_{n i} X_{n i}\right|>\varepsilon b_{n}\right) \subset\left(\max _{1 \leq i \leq n}\left|X_{n i}\right|>b_{n}\right) \bigcup\left(\max _{1 \leq j \leq n}\left|\sum_{i=1}^{j} a_{n i} X_{i}^{(n)}\right|>\varepsilon b_{n}\right),
$$

which implies that

$$
\begin{aligned}
& P\left(\max _{1 \leq j \leq n}\left|\sum_{i=1}^{j} a_{n i} X_{n i}\right|>\varepsilon b_{n}\right) \\
& \quad \leq P\left(\max _{1 \leq i \leq n}\left|X_{n i}\right|>b_{n}\right)+P\left(\max _{1 \leq j \leq n}\left|\sum_{i=1}^{j} a_{n i} X_{i}^{(n)}\right|>\varepsilon b_{n}\right) \\
& \quad \leq \sum_{i=1}^{n} P\left(\left|X_{n i}\right|>b_{n}\right)+P\left(\max _{1 \leq j \leq n}\left|T_{j}^{(n)}\right|>\varepsilon b_{n}-\max _{1 \leq j \leq n}\left|\sum_{i=1}^{j} a_{n i} E X_{i}^{(n)}\right|\right) .
\end{aligned}
$$


Firstly, we will show that

$$
b_{n}^{-1} \max _{1 \leq j \leq n}\left|\sum_{i=1}^{j} a_{n i} E X_{i}^{(n)}\right| \longrightarrow 0, \quad \text { as } n \longrightarrow \infty .
$$

By $\sum_{i=1}^{n}\left|a_{n i}\right|^{\alpha}=O\left(n^{\delta}\right)$ and Hölder's inequality, we have for $1 \leq k<\alpha$ that

$$
\sum_{i=1}^{n}\left|a_{n i}\right|^{k} \leq\left(\sum_{i=1}^{n}\left(\left|a_{n i}\right|^{k}\right)^{\alpha / k}\right)^{k / \alpha}\left(\sum_{i=1}^{n} 1\right)^{(\alpha-k) / \alpha} \leq C n
$$

Hence, when $1<\alpha<2$, we have by $E X_{n i}=0$, (1.6) of Lemma 1.6, (2.7) (taking $k=1$ ), Markov's inequality, and (2.1) that

$$
\begin{aligned}
b_{n}^{-1} \max _{1 \leq j \leq n}\left|\sum_{i=1}^{j} a_{n i} E X_{i}^{(n)}\right| & \leq \sum_{i=1}^{n}\left|a_{n i}\right| P\left(\left|X_{n i}\right|>b_{n}\right)+b_{n}^{-1} \max _{1 \leq j \leq n}\left|\sum_{i=1}^{j} a_{n i} E X_{n i} I\left(\left|X_{n i}\right|>b_{n}\right)\right| \\
& \leq C \sum_{i=1}^{n}\left|a_{n i}\right| P\left(|X|>b_{n}\right)+b_{n}^{-1} \sum_{i=1}^{n}\left|a_{n i}\right| E\left|X_{n i}\right| I\left(\left|X_{n i}\right|>b_{n}\right) \\
& \leq C n \frac{E \exp \left(h|X|^{\gamma}\right)}{\exp \left(h b_{n}^{\gamma}\right)}+C b_{n}^{-1} \sum_{i=1}^{n}\left|a_{n i}\right| E|X| I\left(|X|>b_{n}\right) \\
& \leq \frac{C n}{n^{h r^{\gamma / \alpha}}}+C b_{n}^{-1} n E|X| I\left(|X|>b_{n}\right) \\
& =\frac{C n}{n^{h r^{\gamma / \alpha}}}+C b_{n}^{-1} n \sum_{k=n}^{\infty} E|X| I\left(b_{k}<|X| \leq b_{k+1}\right) \\
& \leq \frac{C n}{n^{h n^{\gamma / \alpha}}}+C b_{n}^{-1} n \sum_{k=n}^{\infty} b_{k+1} P\left(|X|>b_{k}\right) \\
& \leq \frac{C n}{n^{h r^{\gamma / \alpha}}}+C b_{n}^{-1} n \sum_{k=n}^{\infty} b_{k+1} \frac{E \exp \left(h|X|^{\gamma}\right)}{\exp \left(h b_{k}^{\gamma}\right)} \\
& \leq \frac{C n}{n^{h r^{\gamma / \alpha}}}+C b_{n}^{-1} n \sum_{k=n}^{\infty}(k+1)^{1 / \alpha}(\log (k+1))^{1 / \gamma} k^{-h k^{\gamma / \alpha}} \\
& \leq \frac{C n}{n^{h n^{\gamma / \alpha}}}+C b_{n}^{-1} \sum_{k=n}^{\infty}(k+1)^{1 / \alpha+1}(\log (k+1))^{1 / \gamma} k^{-h k^{\gamma / \alpha}} \\
& \leq \frac{C n}{n^{h n^{\gamma / \alpha}}}+C n^{-1 / \alpha}(\log n)^{-1 / \gamma} \longrightarrow 0, \text { as } n \longrightarrow \infty .
\end{aligned}
$$

Elementary Jensen's inequality implies that for any $0<s<t$,

$$
\left(\sum_{i=1}^{n}\left|a_{n i}\right|^{t}\right)^{1 / t} \leq\left(\sum_{i=1}^{n}\left|a_{n i}\right|^{s}\right)^{1 / s}
$$


Therefore, when $0<\alpha \leq 1$, we have by (1.5) of Lemma 1.6, (2.9), Markov's inequality, and (2.1) that

$$
\begin{aligned}
b_{n}^{-1} \max _{1 \leq j \leq n}\left|\sum_{i=1}^{j} a_{n i} E X_{i}^{(n)}\right| \leq & \sum_{i=1}^{n}\left|a_{n i}\right| P\left(\left|X_{n i}\right|>b_{n}\right)+b_{n}^{-1} \sum_{i=1}^{n}\left|a_{n i}\right| E\left|X_{n i}\right| I\left(\left|X_{n i}\right| \leq b_{n}\right) \\
\leq & C \sum_{i=1}^{n}\left|a_{n i}\right| P\left(|X|>b_{n}\right) \\
& +C b_{n}^{-1} \sum_{i=1}^{n}\left|a_{n i}\right|\left(E|X| I\left(|X| \leq b_{n}\right)+b_{n} P\left(|X|>b_{n}\right)\right) \\
\leq & C b_{n}^{-1} n^{\delta / \alpha} E|X| I\left(|X| \leq b_{n}\right)+C n^{\delta / \alpha} P\left(|X|>b_{n}\right) \\
\leq & C b_{n}^{-1} n^{\delta / \alpha} \sum_{k=2}^{n} E|X| I\left(b_{k-1}<|X| \leq b_{k}\right)+\frac{C n^{\delta / \alpha} E \exp \left(h|X|^{\gamma}\right)}{\exp \left(h b_{n}^{\gamma}\right)} \\
\leq & C b_{n}^{-1} n^{\delta / \alpha} \sum_{k=2}^{n} b_{k} P\left(|X|>b_{k-1}\right)+\frac{C n^{\delta / \alpha}}{n^{h n^{\gamma / \alpha}}} \\
\leq & C b_{n}^{-1} n^{\delta / \alpha} \sum_{k=2}^{n} b_{k} \frac{E \exp \left(h|X|^{\gamma}\right)}{\exp \left(h b_{k-1}^{\gamma}\right)}+\frac{C n^{\delta / \alpha}}{n^{h n^{\gamma / \alpha}}} \\
\leq & C b_{n}^{-1} n^{\delta / \alpha} \sum_{k=2}^{n} k^{1 / \alpha}(\log k)^{1 / \gamma}(k-1)^{-h(k-1)^{\gamma / \alpha}}+\frac{C n^{\delta / \alpha}}{n^{h n^{\gamma / \alpha}}} \\
\leq & C n^{-1 / \alpha}(\log n)^{-1 / \gamma} n^{\delta / \alpha}+\frac{C n^{\delta / \alpha}}{n^{h n^{\gamma / \alpha}}} \\
= & C(\log n)^{-1 / \gamma} n^{\delta / \alpha-1 / \alpha}+\frac{C n^{\delta / \alpha}}{n^{h \eta^{\gamma / \alpha}}} \longrightarrow 0, \text { as } n \longrightarrow \infty .
\end{aligned}
$$

By (2.8) and (2.10), we can get (2.6) immediately. Hence, for $n$ large enough,

$$
P\left(\max _{1 \leq j \leq n}\left|\sum_{i=1}^{j} a_{n i} X_{n i}\right|>\varepsilon b_{n}\right) \leq \sum_{i=1}^{n} P\left(\left|X_{n i}\right|>b_{n}\right)+P\left(\max _{1 \leq j \leq n}\left|T_{j}^{(n)}\right|>\frac{\varepsilon}{2} b_{n}\right) .
$$

To prove (2.2), we only need to show that

$$
\begin{gathered}
I \doteq \sum_{n=1}^{\infty} n^{s \alpha-2} \sum_{i=1}^{n} P\left(\left|X_{n i}\right|>b_{n}\right)<\infty, \\
J \doteq \sum_{n=1}^{\infty} n^{s \alpha-2} P\left(\max _{1 \leq j \leq n}\left|T_{j}^{(n)}\right|>\frac{\varepsilon}{2} b_{n}\right)<\infty .
\end{gathered}
$$


By Definition 1.3, Markov's inequality and (2.1), we can see that

$$
\begin{aligned}
I & \doteq \sum_{n=1}^{\infty} n^{s \alpha-2} \sum_{i=1}^{n} P\left(\left|X_{n i}\right|>b_{n}\right) \\
& \leq C \sum_{n=1}^{\infty} n^{s \alpha-2} \sum_{i=1}^{n} P\left(|X|>b_{n}\right) \\
& \leq C \sum_{n=1}^{\infty} n^{s \alpha-1} \frac{E \exp \left(h|X|^{\gamma}\right)}{\exp \left(h b_{n}^{\gamma}\right)} \\
& \leq C \sum_{n=1}^{\infty} \frac{n^{s \alpha-1}}{n^{h n^{\gamma / \alpha}}}<\infty .
\end{aligned}
$$

For fixed $n \geq 1$, it is easily seen that $\left\{X_{i}^{(n)}, 1 \leq i \leq n\right\}$ are still AANA random variables by Lemma 1.4. For $r>2$, it follows from Lemma 1.5, $C_{r}$ 's inequality, and Jensen's inequality that

$$
\begin{aligned}
J & \doteq \sum_{n=1}^{\infty} n^{s \alpha-2} P\left(\max _{1 \leq j \leq n}\left|T_{j}^{(n)}\right|>\frac{\varepsilon}{2} b_{n}\right) \\
& \leq C \sum_{n=2}^{\infty} n^{s \alpha-2} b_{n}^{-r} E\left(\max _{1 \leq j \leq n}\left|T_{j}^{(n)}\right|^{r}\right) \\
& \leq C \sum_{n=2}^{\infty} n^{s \alpha-2} b_{n}^{-r}\left[\sum_{i=1}^{n}\left|a_{n i}\right|^{r} E\left|X_{i}^{(n)}-E X_{i}^{(n)}\right|^{r}+\left(\sum_{i=1}^{n}\left|a_{n i}\right|^{2} E\left|X_{i}^{(n)}-E X_{i}^{(n)}\right|^{2}\right)^{r / 2}\right] \\
& \leq C \sum_{n=2}^{\infty} n^{s \alpha-2} b_{n}^{-r} \sum_{i=1}^{n}\left|a_{n i}\right|^{r} E\left|X_{i}^{(n)}\right|^{r}+C \sum_{n=2}^{\infty} n^{s \alpha-2} b_{n}^{-r}\left(\sum_{i=1}^{n}\left|a_{n i}\right|^{2} E\left|X_{i}^{(n)}\right|^{2}\right)^{r / 2} \\
& \doteq J_{1}+J_{2} .
\end{aligned}
$$

Taking $r>\max \{2, \alpha(s \alpha-1) /(1-\delta)\}$, which implies that $r>\alpha$. It follows from $C_{r}$ 's inequality, (1.5) of Lemma 1.6, (2.9), Markov's inequality, and (2.1) that

$$
\begin{aligned}
J_{1} & \doteq C \sum_{n=2}^{\infty} n^{s \alpha-2} b_{n}^{-r} \sum_{i=1}^{n}\left|a_{n i}\right|^{r} E\left|X_{i}^{(n)}\right|^{r} \\
& \leq C \sum_{n=2}^{\infty} n^{s \alpha-2} b_{n}^{-r} \sum_{i=1}^{n}\left|a_{n i}\right|^{r}\left[E\left|X_{n i}\right|^{r} I\left(\left|X_{n i}\right| \leq b_{n}\right)+b_{n}^{r} P\left(\left|X_{n i}\right|>b_{n}\right)\right] \\
& \leq C \sum_{n=2}^{\infty} n^{s \alpha-2} b_{n}^{-r} \sum_{i=1}^{n}\left|a_{n i}\right|^{r}\left[E|X|^{r} I\left(|X| \leq b_{n}\right)+b_{n}^{r} P\left(|X|>b_{n}\right)\right] \\
& \leq C \sum_{n=2}^{\infty} n^{s \alpha-2+(r \delta / \alpha)} b_{n}^{-r} E|X|^{r} I\left(|X| \leq b_{n}\right)+C \sum_{n=2}^{\infty} n^{s \alpha-2+(r \delta / \alpha)} P\left(|X|>b_{n}\right) \\
& \leq C \sum_{n=2}^{\infty} n^{s \alpha-2+(r \delta / \alpha)} b_{n}^{-r} \sum_{k=2}^{n} E|X|^{\gamma} I\left(b_{k-1}<|X| \leq b_{k}\right)+C \sum_{n=2}^{\infty} n^{s \alpha-2+r \delta / \alpha} \frac{E \exp \left(h|X|^{r}\right)}{\exp \left(h b_{n}^{r}\right)} \\
& \leq C \sum_{k=2}^{\infty} \sum_{n=k}^{\infty} n^{s \alpha-2(r \delta / \alpha)} n^{-r / \alpha}(\log n)^{-r / \gamma} b_{k}^{r} P\left(|X|>b_{k-1}\right)+C \sum_{n=2}^{\infty} \frac{n^{s \alpha-2+(r \delta / \alpha)}}{n^{h n^{\gamma / \alpha}}}
\end{aligned}
$$




$$
\begin{aligned}
& \leq C \sum_{k=2}^{\infty} b_{k}^{r} \frac{E \exp \left(h|X|^{\gamma}\right)}{\exp \left(h b_{k-1}^{\gamma}\right)}+C \sum_{n=2}^{\infty} \frac{n^{s \alpha-2+(r \delta / \alpha)}}{n^{h r^{\gamma / \alpha}}} \\
& \leq C \sum_{k=2}^{\infty} \frac{k^{r / \alpha}(\log k)^{r / \gamma}}{(k-1)^{h(k-1)^{\gamma / \alpha}}+C \sum_{n=2}^{\infty} \frac{n^{s \alpha-2+(r \delta / \alpha)}}{n^{h n^{\gamma / \alpha}}}<\infty .} .
\end{aligned}
$$

By $C_{r}$ 's inequality, (1.5) of Lemma 1.6, (2.9), and Jensen's inequality, we can get that

$$
\begin{aligned}
J_{2} & \doteq C \sum_{n=2}^{\infty} n^{s \alpha-2} b_{n}^{-r}\left(\sum_{i=1}^{n}\left|a_{n i}\right|^{2} E\left|X_{i}^{(n)}\right|^{2}\right)^{r / 2} \\
& \leq C \sum_{n=2}^{\infty} n^{s \alpha-2} b_{n}^{-r}\left(\sum_{i=1}^{n}\left|a_{n i}\right|^{2}\left[E\left|X_{n i}\right|^{2} I\left(\left|X_{n i}\right| \leq b_{n}\right)+b_{n}^{2} P\left(\left|X_{n i}\right|>b_{n}\right)\right]\right)^{r / 2} \\
& \leq C \sum_{n=2}^{\infty} n^{s \alpha-2} b_{n}^{-r}\left[\sum_{i=1}^{n}\left|a_{n i}\right|^{2}\left[E X^{2} I\left(|X| \leq b_{n}\right)+b_{n}^{2} P\left(|X|>b_{n}\right)\right]\right]^{r / 2} \\
& \leq C \sum_{n=2}^{\infty} n^{s \alpha-2+(r \delta / \alpha)} b_{n}^{-r}\left[E X^{2} I\left(|X| \leq b_{n}\right)+b_{n}^{2} P\left(|X|>b_{n}\right)\right]^{r / 2} \\
& \leq C \sum_{n=2}^{\infty} n^{s \alpha-2+(r \delta / \alpha)} b_{n}^{-r}\left[E X^{2} I\left(|X| \leq b_{n}\right)\right]^{r / 2}+C \sum_{n=2}^{\infty} n^{s \alpha-2+(r \delta / \alpha)}\left[P\left(|X|>b_{n}\right)\right]^{r / 2} \\
& \leq C \sum_{n=2}^{\infty} n^{s \alpha-2+(r \delta / \alpha)} b_{n}^{-r} E|X|^{r} I\left(|X| \leq b_{n}\right)+C \sum_{n=2}^{\infty} n^{s \alpha-2+(r \delta / \alpha)} P\left(|X|>b_{n}\right) \\
& <\infty \quad \text { (see the proof of }(2.15)) .
\end{aligned}
$$

Therefore, the desired result (2.2) follows from (2.13)-(2.16) immediately. This completes the proof of the theorem.

Similar to the proof of Theorem 2.1, we can get the following result for sequences of AANA random variables.

Theorem 2.2. Let $\left\{X_{n}, n \geq 1\right\}$ be a sequence of AANA random variables which is stochastically dominated by a random variable $X$, and let $\left\{a_{n i}, i \geq 1, n \geq 1\right\}$ be an array of real numbers. Suppose that the conditions $\left(H_{1}\right)-\left(H_{3}\right)$ are satisfied $\left(E X_{n i}=0\right.$ is replaced by $E X_{n}=0$ in $\left.H_{1}\right)$. Then, for any $\varepsilon>0$,

$$
\sum_{n=1}^{\infty} n^{s \alpha-2} P\left(\max _{1 \leq j \leq n}\left|\sum_{i=1}^{j} a_{n i} X_{i}\right|>\varepsilon b_{n}\right)<\infty,
$$

where $s \geq 1 / \alpha$ and $b_{n} \doteq n^{1 / \alpha} \log ^{1 / \gamma} n$.

The following result provides the Marcinkiewicz-Zygmund type strong law of large numbers for weighted sums $\sum_{i=1}^{n} a_{i} X_{i}$ of AANA sequence of random variables. 
Theorem 2.3. Let $\left\{X_{n}, n \geq 1\right\}$ be a sequence of AANA random variables which is stochastically dominated by a random variable $X$ and $\left\{a_{n}, n \geq 1\right\}$ be a sequence of real numbers. Suppose that the conditions $\left(\mathrm{H}_{2}\right)-\left(\mathrm{H}_{4}\right)$ are satisfied. Then for any $\varepsilon>0$,

$$
\begin{gathered}
\sum_{n=1}^{\infty} n^{s \alpha-2} P\left(\max _{1 \leq j \leq n}\left|S_{j}\right|>\varepsilon b_{n}\right)<\infty, \\
\lim _{n \rightarrow \infty} \frac{S_{n}}{b_{n}}=0 \quad \text { a.s., }
\end{gathered}
$$

where $s \geq 1 / \alpha, b_{n} \doteq n^{1 / \alpha} \log ^{1 / \gamma} n$ and $S_{n}=\sum_{i=1}^{n} a_{i} X_{i}$ for $n \geq 1$.

Proof. Similar to the proof of Theorem 2.1, we can get (2.18) immediately, which yields that

$$
\sum_{n=1}^{\infty} n^{-1} P\left(\max _{1 \leq j \leq n}\left|S_{j}\right|>\varepsilon b_{n}\right)<\infty
$$

Therefore,

$$
\begin{aligned}
\infty & >\sum_{n=1}^{\infty} n^{-1} P\left(\max _{1 \leq j \leq n}\left|S_{j}\right|>\varepsilon b_{n}\right) \\
& =\sum_{i=0}^{\infty} \sum_{n=2^{i}}^{2^{i+1}-1} n^{-1} P\left(\max _{1 \leq j \leq n}\left|S_{j}\right|>\varepsilon n^{1 / \alpha}(\log n)^{1 / \gamma}\right) \\
& \geq \frac{1}{2} \sum_{i=1}^{\infty} P\left(\max _{1 \leq j \leq 2^{i}}\left|S_{j}\right|>\varepsilon 2^{(i+1) / \alpha}\left(\log 2^{i+1}\right)^{1 / \gamma}\right) .
\end{aligned}
$$

By Borel-Cantelli lemma, we obtain that

$$
\lim _{i \rightarrow \infty} \frac{\max _{1 \leq j \leq 2^{i}}\left|S_{j}\right|}{2^{(i+1) / \alpha}\left(\log 2^{i+1}\right)^{1 / \gamma}}=0 \quad \text { a.s. }
$$

For all positive integers $n$, there exists a positive integer $i_{0}$ such that $2^{i_{0}-1} \leq n<2^{i_{0}}$. We have by (2.22) that

$$
\frac{\left|S_{n}\right|}{b_{n}} \leq \max _{2^{i_{0}-1} \leq n<2^{i_{0}}} \frac{\left|S_{n}\right|}{b_{n}} \leq \frac{2^{2 / \alpha} \max _{1 \leq j \leq 2^{i}}\left|S_{j}\right|}{2^{\left(i_{0}+1\right) / \alpha}\left(\log 2^{i_{0}+1}\right)^{1 / \gamma}}\left(\frac{i_{0}+1}{i_{0}-1}\right)^{1 / \gamma} \rightarrow 0 \quad \text { a.s., as } i_{0} \longrightarrow \infty,
$$

which implies (2.19). This completes the proof of the theorem.

Remark 2.4. In Theorems 2.1-2.3, the condition $\left(\mathrm{H}_{1}\right)$ or $\left(\mathrm{H}_{4}\right)$ is needed. Under the weaker condition $\left(\left(\mathrm{H}_{5}\right)\right.$ or $\left.\left(\mathrm{H}_{6}\right)\right)$ than $\left(\left(\mathrm{H}_{1}\right)\right.$ or $\left.\left(\mathrm{H}_{4}\right)\right)$, we can get the following Theorems 2.5-2.7. The details of their proofs are omitted. 
Theorem 2.5. Let $\left\{X_{n i}: i \geq 1, n \geq 1\right\}$ be an array of rowwise AANA random variables which is stochastically dominated by a random variable $X$, and let $\left\{a_{n i}: i \geq 1, n \geq 1\right\}$ be an array of real numbers. Suppose that the conditions $\left(H_{2}\right),\left(H_{3}\right)$, and $\left(H_{5}\right)$ are satisfied. Then, for any $\varepsilon>0$,

$$
\sum_{n=1}^{\infty} n^{-1} P\left(\max _{1 \leq j \leq n}\left|\sum_{i=1}^{j} a_{n i} X_{n i}\right|>\varepsilon b_{n}\right)<\infty
$$

where $b_{n} \doteq n^{1 / \alpha} \log ^{1 / \gamma} n$.

Theorem 2.6. Let $\left\{X_{n}, n \geq 1\right\}$ be a sequence of AANA random variables which is stochastically dominated by a random variable $X$, and let $\left\{a_{n i}, i \geq 1, n \geq 1\right\}$ be an array of real numbers. Suppose that the conditions $\left(H_{2}\right),\left(H_{3}\right)$ and $\left(H_{5}\right)$ are satisfied $\left(E X_{n i}=0\right.$ is replaced by $E X_{n}=0$ in $\left.\left(H_{5}\right)\right)$. Then, for any $\varepsilon>0$,

$$
\sum_{n=1}^{\infty} n^{-1} P\left(\max _{1 \leq j \leq n}\left|\sum_{i=1}^{j} a_{n i} X_{i}\right|>\varepsilon b_{n}\right)<\infty
$$

where $b_{n} \doteq n^{1 / \alpha} \log ^{1 / \gamma} n$.

Theorem 2.7. Let $\left\{X_{n}, n \geq 1\right\}$ be a sequence of AANA random variables which is stochastically dominated by a random variable $X$, and let $\left\{a_{n}, n \geq 1\right\}$ be a sequence of real numbers. Suppose that the conditions $\left(\mathrm{H}_{2}\right),\left(\mathrm{H}_{3}\right)$, and $\left(\mathrm{H}_{6}\right)$ are satisfied. Then, for any $\varepsilon>0$,

$$
\begin{gathered}
\sum_{n=1}^{\infty} n^{-1} P\left(\max _{1 \leq j \leq n}\left|S_{j}\right|>\varepsilon b_{n}\right)<\infty, \\
\lim _{n \rightarrow \infty} \frac{S_{n}}{b_{n}}=0 \quad \text { a.s., }
\end{gathered}
$$

where $b_{n} \doteq n^{1 / \alpha} \log ^{1 / \gamma} n$ and $S_{n}=\sum_{i=1}^{n} a_{i} X_{i}$ for $n \geq 1$.

\section{Acknowledgments}

The authors are most grateful to the Editor Wei-Der Chang and anonymous referees for careful reading of the paper and valuable suggestions which helped in improving an earlier version of this paper. This work was supported by the NNSF of China (11171001), Provincial Natural Science Research Project of Anhui Colleges (KJ2010A005), Talents Youth Fund of Anhui Province Universities (2010SQRL016ZD), Youth Science Research Fund of Anhui University (2009QN011A), and the academic innovation team of Anhui University (KJTD001B).

\section{References}

[1] P. L. Hsu and H. Robbins, "Complete convergence and the law of large numbers," Proceedings of the National Academy of Sciences of the United States of America, vol. 33, pp. 25-31, 1947.

[2] K. Joag-Dev and F. Proschan, "Negative association of random variables, with applications," The Annals of Statistics, vol. 11, no. 1, pp. 286-295, 1983. 
[3] P. Matuła, "A note on the almost sure convergence of sums of negatively dependent random variables," Statistics \& Probability Letters, vol. 15, no. 3, pp. 209-213, 1992.

[4] T. K. Chandra and S. Ghosal, "Extensions of the strong law of large numbers of Marcinkiewicz and Zygmund for dependent variables," Acta Mathematica Hungarica, vol. 71, no. 4, pp. 327-336, 1996.

[5] T. K. Chandra and S. Ghosal, "The strong law of large numbers for weighted averages under dependence assumptions," Journal of Theoretical Probability, vol. 9, no. 3, pp. 797-809, 1996.

[6] M.-H. Ko, T.-S. Kim, and Z. Lin, "The Hajeck-Rènyi inequality for the AANA random variables and its applications," Taiwanese Journal of Mathematics, vol. 9, no. 1, pp. 111-122, 2005.

[7] Y. Wang, J. Yan, F. Cheng, and C. Su, "The strong law of large numbers and the law of the iterated logarithm for product sums of NA and AANA random variables," Southeast Asian Bulletin of Mathematics, vol. 27, no. 2, pp. 369-384, 2003.

[8] D. Yuan and J. An, "Rosenthal type inequalities for asymptotically almost negatively associated random variables and applications," Science in China. Series A: Mathematics, vol. 52, no. 9, pp. 1887$1904,2009$.

[9] X. J. Wang, S. H. Hu, and W. Z. Yang, "Convergence properties for asymptotically almost negatively associated sequence," Discrete Dynamics in Nature and Society, vol. 2010, Article ID 218380, 15 pages, 2010 . 


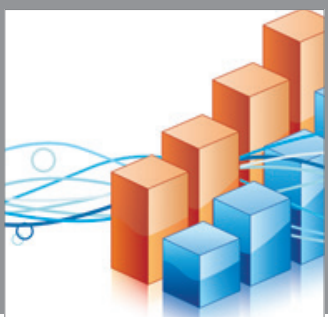

Advances in

Operations Research

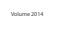

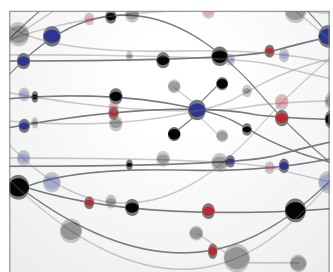

\section{The Scientific} World Journal
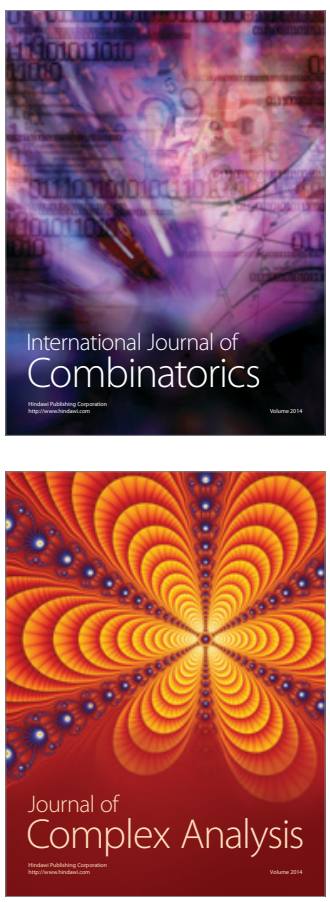

International Journal of

Mathematics and

Mathematical

Sciences
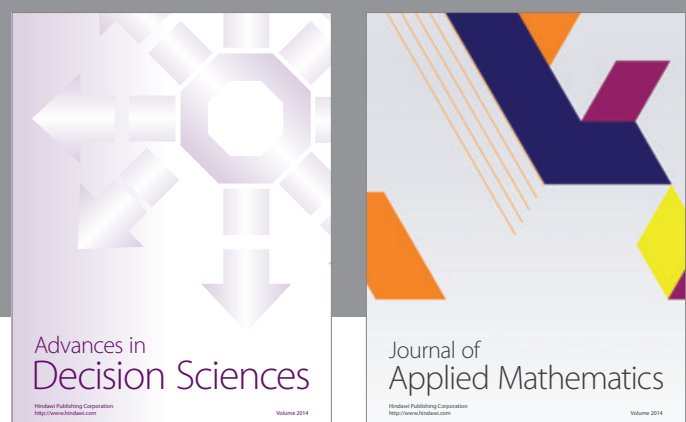

Journal of

Applied Mathematics
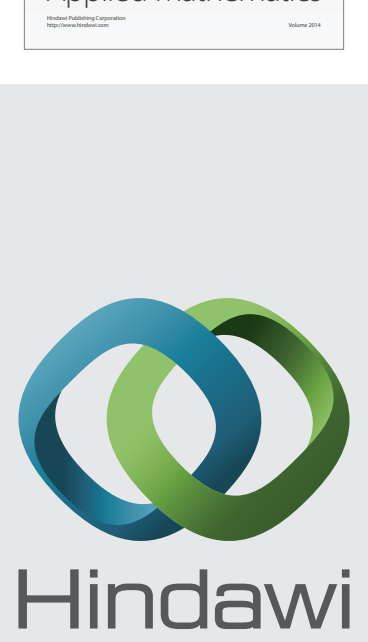

Submit your manuscripts at http://www.hindawi.com
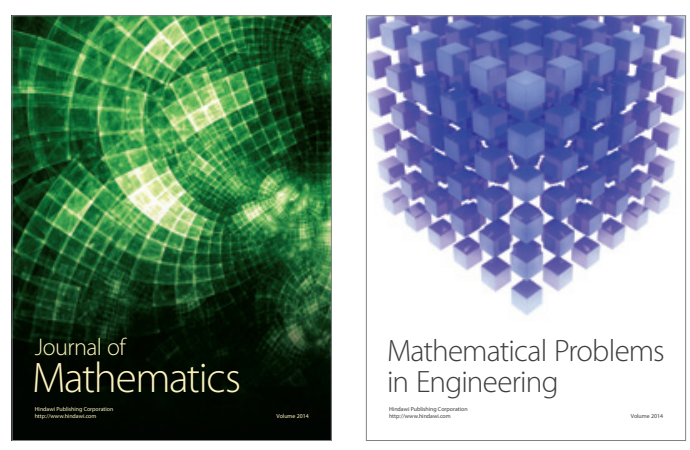

Mathematical Problems in Engineering
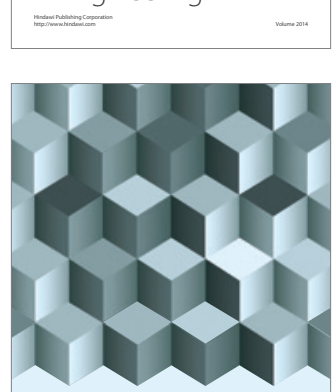

Journal of

Function Spaces
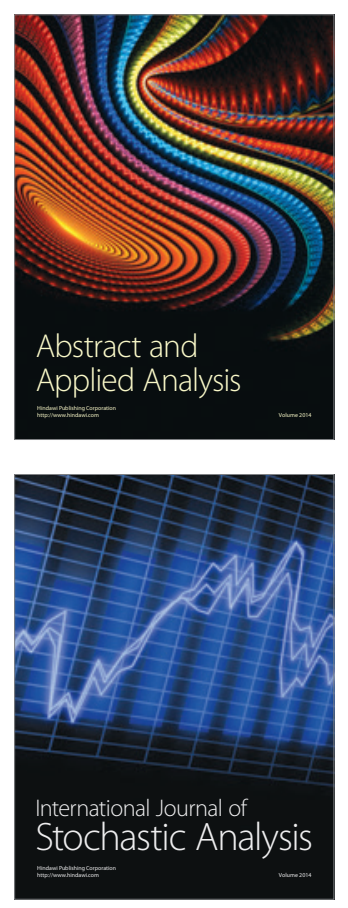

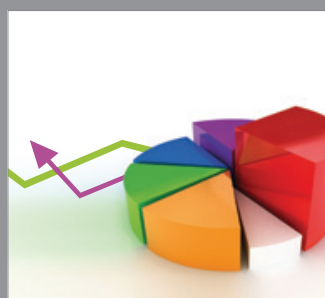

ournal of

Probability and Statistics

Promensencen
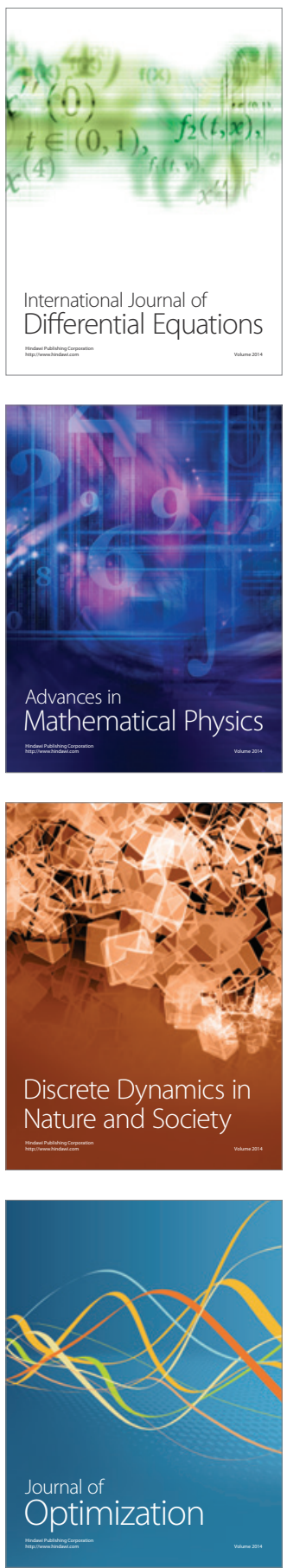